\title{
Characterization and genetic mapping of eceriferum-ym (cer-ym), a cutin deficient barley mutant with impaired leaf water retention capacity
}

\author{
Chao Li ${ }^{1,2)}$, Cheng Liu ${ }^{2)}$, Xiaoying Ma'), Aidong Wang'), Ruijun Duan'), Christiane Nawrath ${ }^{3)}$, \\ Takao Komatsuda ${ }^{2}$ and Guoxiong Chen ${ }^{* 1,2)}$ \\ 1) Laboratory of Plant Stress Ecophysiology and Biotechnology, Cold and Arid Regions Environmental and Engineering Research Insti- \\ tute, Chinese Academy of Sciences, Lanzhou 730000, China \\ 2) National Institute of Agrobiological Sciences, Tsukuba, Ibaraki 305-8602, Japan \\ 3) Department of Plant Molecular Biology, University of Lausanne, Lausanne CH-1015, Switzerland
}

The cuticle covers the aerial parts of land plants, where it serves many important functions, including water retention. Here, a recessive cuticle mutant, eceriferum-ym (cer-ym), of Hordeum vulgare L. (barley) showed abnormally glossy spikes, sheaths, and leaves. The cer-ym mutant plant detached from its root system was hypersensitive to desiccation treatment compared with wild type plants, and detached leaves of mutant lost $41.8 \%$ of their initial weight after $1 \mathrm{~h}$ of dehydration under laboratory conditions, while that of the wild type plants lost only $7.1 \%$. Stomata function was not affected by the mutation, but the mutant leaves showed increased cuticular permeability to water, suggesting a defective leaf cuticle, which was confirmed by toluidine blue staining. The mutant leaves showed a substantial reduction in the amounts of the major cutin monomers and a slight increase in the main wax component, suggesting that the enhanced cuticle permeability was a consequence of cutin deficiency. cer-ym was mapped within a $0.8 \mathrm{cM}$ interval between EST marker AK370363 and AK251484, a pericentromeric region on chromosome $4 \mathrm{H}$. The results indicate that the desiccation sensitivity of cer-ym is caused by a defect in leaf cutin, and that cer-ym is located in a chromosome $4 \mathrm{H}$ pericentromeric region.

Key Words: eceriferum-mutant, cuticle, cutin, desiccation resistance, genetic mapping, wax.

\section{Introduction}

Water availability is one of the most critical requirements for the survival of terrestrial plants and water deficit is a common cause of decreased productivity and restricted growth (Boyer 1982). In this regard, the formation of an extracellular hydrophobic cuticle, which coats the outer surfaces of land plant aerial organs, thereby limiting water loss, has been a crucial evolutionary innovation (Goodwin and Jenks 2005). In addition, the cuticle provides protection against a range of abiotic and biotic stresses and has an important influence on plant growth and development (Yeats and Rose 2013).

The structure and properties of the cuticle vary considerably among plant species, organs and growth stages, but it mainly consists of two hydrophobic components, cutin and waxes (Jeffree 1996). The cutin matrix is a polyester composed of inter-esterified oxygenated $\mathrm{C} 16$ and $\mathrm{C} 18$ fatty

Communicated by N. Stein

Received March 10, 2015. Accepted June 24, 2015.

*Corresponding author (e-mail: guoxiong@1zb.ac.cn) acids and glycerol, and typically comprises $40-80 \%$ of the cuticular weight (Heredia 2003, Nawrath 2006). Waxes embedded in the cutin matrix (intracuticular) or deposited on its surface (epicuticular) are mostly composed of an aliphatic mixture of very long chain (VLC) fatty acids and their derivatives, including alkanes, alcohols, aldehydes, ketones and alkyl esters (Buschhaus and Jetter 2011, Nawrath 2006). The cutin matrix thus provides the framework for wax deposition and its structural organization and size may affect the fine structure and water-barrier functions of the waxes. Unlike the distinct patterns of regulation associated with different wax components, a study of Arabidopsis thaliana leaf cuticles revealed an indiscriminate increase in the abundance of all cutin monomers by $65 \%$ following exposure to water stress (Kosma et al. 2009). This may suggest that the total amount of polymeric cutin is more important for water retention than the relative proportion of any specific cutin monomer. It has been demonstrated that the transcription factor WIN1/SHN1, an important regulator of cutin synthesis, controls cuticular water permeability by directly targeting the long-chain acyl-CoA synthetase 2 (LACS2), which is responsible for the synthesis of cutin 
intermediates (Kannangara et al. 2007). Moreover, cutin deficiency has been associated with substantial increases in the permeability of cuticles that show no major qualitative or quantitative changes in the constituent waxes in a number of mutants, such as the A. thaliana double mutant glycerol3-phosphate acyltransferase 4 (gpat4)/gpat8 (Li et al. 2007), defective cuticular ridges (dcr) (Panikashvili et al. 2009), the Oryza sativa (rice) wilted dwarf and lethal 1 (wdl1) (Park et al. 2010) and the Solanum lycopersicum (tomato) cutin deficient 1 (cdl) mutant (Isaacson et al. 2009) and the Hordeum vulgare (barley) mutants eibil (Chen et al. 2011) and eceriferum-zv (cer-zv) (Li et al. 2013).

More than 20 genes that participate in cutin synthesis, transportation, polymerization and regulation have been identified and characterized (Yeats and Rose 2013), most of which are from A. thaliana. However, other plant species are providing valuable experimental systems, such as barley, where a collection of 1,580 cuticle eceriferum (cer) mutants with glossy leaves, sheaths and/or spikes have been generated as a consequence of chemical and physical mutagenesis, and the underling mutations have been ascribed to 79 distinct genetic loci (Lundqvist and Lundqvist 1988). Some of these loci have been roughly mapped (Druka et al. 2011, Franckowiak 1997), but none of the mutated genes has been cloned or fine mapped to date. The single recessive cer mutants eceriferum-ym (cer-ym) and cer-zv fall into the category of plants with glossy spikes, sheaths and leaves (Franckowiak and Lundqvist 2012). The cer-ym and cer-zv mutations have been localized to similar genomic regions, $72.70-101.44 \mathrm{cM}$ and $47.80-107.76 \mathrm{cM}$, respectively, on chromosome 4H (Druka et al. 2011).

In this study, we report the characterization of cer-ym and the genetic mapping of cer-ym locus, and we report that cer-ym is a cutin defective mutant.

\section{Materials and Methods}

\section{Plant materials}

The barley mutant line cer-ym.753 was originally induced in the cultivar 'Bonus' by ethylene oxide (Franckowiak and Lundqvist 2012). Seeds of Bowman-Near isogenic line carrying cer-ym.753 (GSHO 2217, $\mathrm{BC}_{4}$ ), named hereafter BW-NIL (cer-ym.753), and Bowman (PI 483237) were obtained from the United States Department of Agriculture, Agricultural Research Service (USDA-ARS) (http://www. ars-grin.gov/npgs/acc/acc_queries.html). The $\mathrm{BC}_{6}$ generation of GSHO 2217 was termed as BW144 in Druka et al. (2011) and not identical with GSHO 2217. Hordeum vulgare subsp. spontaneum accession 'OUH602' (wild barley) was obtained from the Institute of Plant Science and Resources, Okayama University, Kurashiki, Japan.

\section{Water loss measurements}

A leaf-drying assay was conducted by sampling $2 \mathrm{~cm}$ sections of fully expanded leaves from BW-NIL (cer$y m .753)$ and Bowman at the one-leaf stage and keeping them under laboratory conditions for $3 \mathrm{~h}$. To test the leaf water loss rate, $8 \mathrm{~cm}$ lateral sections of fully expanded leaves were collected from BW-NIL (cer-ym.753) and Bowman at the end of the tillering stage, and kept under laboratory conditions. Leaf weight was recorded at $30 \mathrm{~min}$ intervals for $2 \mathrm{~h}$ and the percentage loss in fresh weight was presented as means $\pm \mathrm{SD}$ for three biological replicates. To compare the water loss at the whole plant level, roots were removed from BW-NIL (cer-ym.753) and Bowman at the end of the tillering stage and the remaining shoot was kept under laboratory conditions for $24 \mathrm{~h}$.

\section{Transpiration rate assay}

Distal $10 \mathrm{~cm}$ sections from fully expanded leaves were collected from BW-NIL (cer-ym.753) and Bowman at the end of the tillering stage. The cut end was immediately immersed in distilled water in a $2 \mathrm{~mL}$ tube and the junction between the leaf sample and the tube was sealed with Parafilm (American National Can). The leaf samples were placed at $26^{\circ} \mathrm{C}, 60 \%$ relative humidity in the light $\left(108 \mu \mathrm{mol}\right.$ quanta s $\left.\mathrm{s}^{-1} \mathrm{~m}^{-2}\right)$ for $3 \mathrm{~h}$, and then in darkness for $6 \mathrm{~h}$. The combined weight of the tube, water and leaf was measured before and after different light conditions and the water loss by transpiration was calculated based on the weight loss. Leaf area was determined by analyzing leaf photographs taken after the treatments using Image J software (National Institutes of Health, Bethesda, MD, USA; http://rsb.info.nih.gov/ij/). The detached-leaf transpiration of each sample was expressed as weight $(\mathrm{g})$ of water loss per unit area $\left(\mathrm{m}^{2}\right)$ per hour $\left(\mathrm{g}\right.$ water $\left.\mathrm{m}^{-2} \mathrm{~h}^{-1}\right)$ and shown as means \pm SD for either three or four biological replicates.

\section{Toluidine blue (TB) test of cuticle permeability}

Approximately $2 \mathrm{~cm}$ long segments from fully expanded leaves of BW-NIL (cer-ym.753) and Bowman at the end of tillering stage were sampled and placed in $0.05 \%$ (weight/ volume) aqueous toluidine blue (TB) stain (Solarbio) at room temperature for $3 \mathrm{~h}$. After washing with distilled water, the leaf segments were imaged with a digital camera (Cannon PC1587).

\section{Cutin monomer and wax analysis}

Cuticular lipids from the middle $6 \mathrm{~cm}$ segments of fully expanded mature leaves of BW-NIL (cer-ym.753) and Bowman at the tillering stage were analyzed by gas chromatography-mass spectrometry/flame ionization detector (GC-MS/FID) and presented as means \pm SD for four biological replicates. The leaf area was calculated using ImageJ software to evaluate digital photographs. Leaf waxes were extracted by immersing the leaf sample in chloroform for $20 \mathrm{~s}$, before drying and derivatization for GC-MS/FID as described by Greer et al. (2007). The cutin components were prepared for GC-MS/FID analysis from the dewaxed samples by extended delipidation, depolymerization, and derivatization with N,O-bis(trimethylsilyl) trifluoroacetamide as described by Bessire et al. (2007). 


\section{Genetic mapping}

A set of $123 \mathrm{~F}_{2}$ plants from the cross OUH $602 \times \mathrm{BW}$ NIL (cer-ym.753) (GSHO 2217) were analyzed by exploiting a set of markers (AK358684, AK370363, AK248269, AK364461, AK251484 and AK364819) flanking the cer-zv locus on chromosome 4H (Li et al. 2013). Segments of detached leaves from the $\mathrm{F}_{3}$ generation (16-20 progenies per $\mathrm{F}_{2}$ line) of the mapping population were phenotyped for the severe-desiccation trait as described in the leaf drying assay. The genotype on cer-ym locus for each $\mathrm{F}_{2}$ line was deduced from the phenotype of its $\mathrm{F}_{3}$ progenies. Linkage map was constructed using Map Maker3 (Lander et al. 1987) and the Kosambi’s mapping function (Kosambi 1944).

\section{Results}

\section{cer-ym is sensitive to desiccation treatment}

The BW-NIL (cer-ym.753) exhibited a semi-dwarf phenotype and a substantial reduction in visible wax crystals in leaf (Fig. 1A), similar to the phenotype of Bowman-near isogenic line carrying cer-zv.268 allele (GSHO 2207, $\mathrm{BC}_{4}$ ), named hereafter BW-NIL (cer-zv.268), which is a known cutin related recessive mutant that has a reduced waterretention capacity in its leaves ( $\mathrm{Li}$ et al. 2013). We therefore hypothesized that the water-retention capacity might be similarly impaired in BW-NIL (cer-ym.753), and this was tested using several different experiments. When detached leaves were placed under laboratory conditions, the mutant leaves exhibited a severely desiccated appearance after $3 \mathrm{~h}$ of dehydration (Fig. 1B). The fresh weight of BW-NIL (cer$y m .753)$ leaves decreased by $41.8 \pm 3.1 \%$ after $1 \mathrm{~h}$ and $69.9 \pm 3.8 \%$ after $2 \mathrm{~h}$ of dehydration, compared to the Bowman leaf weight that only decreased by $7.1 \pm 0.8 \%$ and $12.7 \pm 1.9 \%$, respectively (Fig. 1C). The BW-NIL (cer$y m .753$ ) shoots from which the roots had been removed at the ending of the tillering stage also showed far more extensive desiccation (Fig. 1D) after $24 \mathrm{~h}$ of dehydration compared to the equivalent Bowman shoots, further indicating the hypersensitivity of cer-ym to desiccation.

\section{cer-ym has a defective leaf cuticle}

The transpiration rates of detached BW-NIL (cer-ym.753) and Bowman leaves were reduced substantially, but to a similar extent, in the absence of light (Fig. 1E), suggesting that the stomata of BW-NIL (cer-ym.753) leaves function normally. Leaf transpiration occurs primarily through the cuticle when stomata are closed in the dark (Niederl et al. 1998), and we observed that BW-NIL (cer-ym.753) leaf transpiration rate were considerably higher than those of Bowman leaves under both dark and light conditions (Fig. 1E), supporting the idea that cer-ym has a defective leaf cuticle. Increased permeability of BW-NIL (cer$y m .753)$ cuticles was further evidenced by strong staining of BW-NIL (cer-ym.753) leaves with TB after a $3 \mathrm{~h}$ of incubation, while Bowman leaves showed not such staining (Fig. 1F).
The cer-ym leaf cuticle shows a major reduction in the levels of cutin monomers

The cutin and waxes of the cuticle of mature leaves at the tillering stage were extracted and analyzed to help establish whether the absence of any particular component might explain the increased cuticle permeability of the cer-ym mutant. The four main cutin monomers in wild type barley leaves are: omega-hydroxy-9, 10-epoxy octadecanoic acid ( $\omega \mathrm{OH}-9,10$ epoxy C18); 9(10), 16-dihydroxyhexadecanoic acid (9(10), 16-OH C16); omega-hydroxyoctadecenoic acid $(\omega \mathrm{OH} \mathrm{C} 18: 1)$; and omega-hydroxyhexadecanoic acid $(\omega \mathrm{OH}$ $\mathrm{C} 16)$. We determined that the levels of all four monomers were substantially reduced $(60 \%-90 \%)$ in BW-NIL (cer$y m .753$ ) leaves compared to Bowman leaves (Fig. 2A). In contrast, the levels of 1-hexacosanol, the main wax component, which is thought to be an important marker for wax deposition, were significantly higher $(\sim 16 \%)$ in BW-NIL (cer-ym.753) leaves compared to Bowman (Fig. 2B). Based on these results we inferred that the increased permeability of BW-NIL (cer-ym.753) leaves is caused by an overall reduction in cutin polymer deposition.

cer-ym and cer-Zv map to the same pericentromeric region

A set of cer-zv locus-flanking markers, positioned in the pericentromeric region of chromosome $4 \mathrm{H}$, were used to locate the cer-ym locus. It was shown that cer-ym mapped within a $0.8 \mathrm{cM}$ region between AK370363 and AK251484, and co-segregated with AK248269 and AK364461 (Fig. 3).

\section{Discussion}

The reduced leaf water-retention capacity of cer-ym can be attributed to a defective cuticle

The cer-ym leaves exhibited a substantially higher transpiration rate regardless of whether their stomata, which functioned normally, were open or closed, and the mutant leaves were more readily stained by TB than those of Bowman plants. In addition, the levels of the main cutin monomers showed a major decrease in the mutant leaves, while the primary wax component was slightly more abundant. Taken together, these results suggest that the reduced capacity of cer-ym leaves to retain water is caused by a severe reduction in the amount of leaf cutin.

The barley cutin mutants eibil and cer-zv have been reported to be hypersensitive to desiccation (Chen et al. 2011, Li et al. 2013). The desiccation-sensitive trait of eibil is associated with a cutin deficiency, which is caused by a lesion in a gene encoding an ABCG31 transporter (Chen et al. 2011). The cer-ym mutant has been thought to be distinct from eibil and to be caused by a mutation at a different locus from eibil (which is located on chromosome $3 \mathrm{H}$ ) (Druka et al. 2011). Indeed, the monomer profiles of the two mutants are different since the main cutin monomer, $\omega \mathrm{OH}-$ 9, 10 epoxy C18, was reported to be reduced by only $50 \%$ in eibil (Chen et al. 2011), but it shows a more substantial decrease ( $90 \%)$ in BW-NIL (cer-ym. 753) (Fig. 2A). 
A

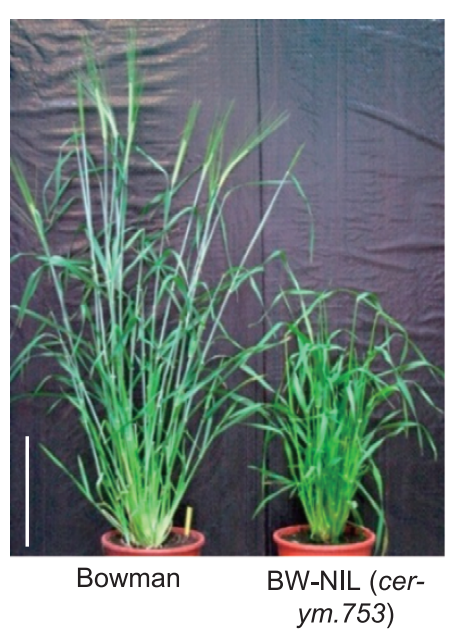

B
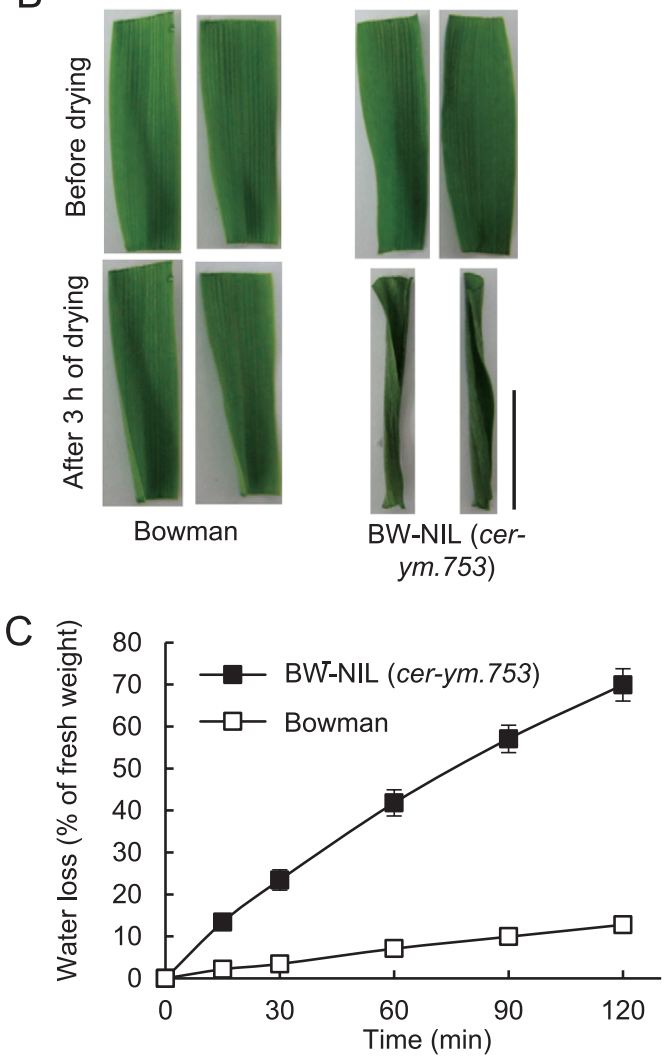

$\mathrm{D}$

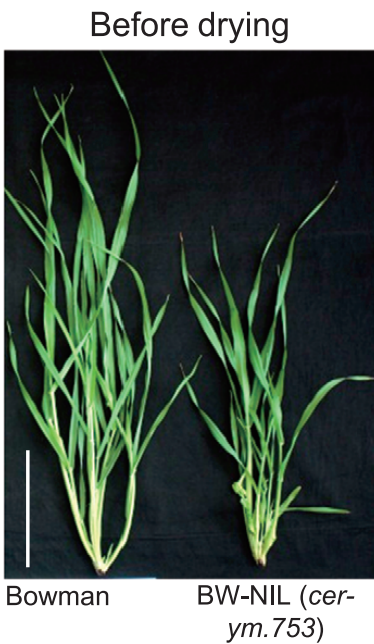

After $24 \mathrm{~h}$ of drying

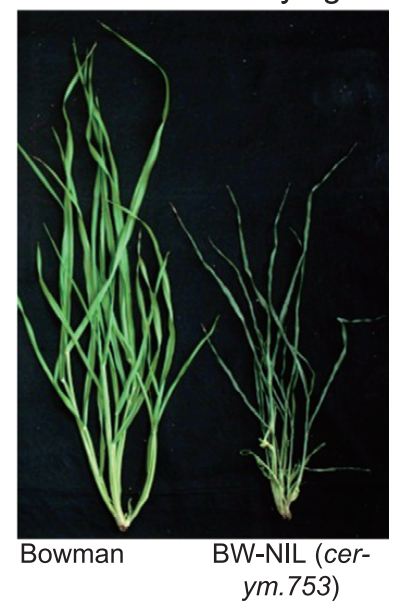

E

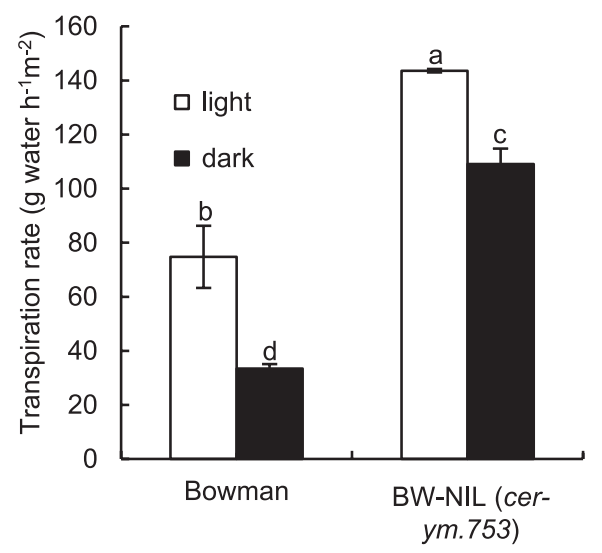

F

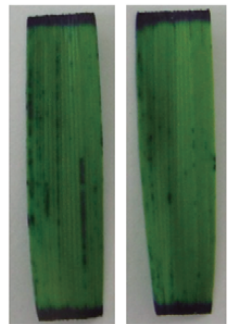

Bowman

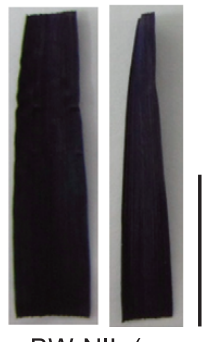

BW-NIL (cerym.753)

Fig. 1. Defective BW-NIL (cer-ym.753) leaf cuticle. (A) Contrasting morphologies of BW-NIL (cer-ym.753) (GSHO 2217) and Bowman. Scale bar, $10 \mathrm{~cm}$. (B) Desiccation sensitivity of BW-NIL (cer-ym.753) and Bowman under laboratory conditions. Detached leaves after $3 \mathrm{~h}$ of drying. Scale bar, $1 \mathrm{~cm}$. (C) Water loss rates of detached leaves. Error bars represent S.D. of biological replicates $(\mathrm{n}=3)$. (D) Plants with the roots removed after $24 \mathrm{~h}$ of drying. Scale bar, $10 \mathrm{~cm}$. (E) Average transpiration rate of detached leaves from BW-NIL (cer-ym.753) and Bowman under light and dark conditions. Different letters denote significant differences at $\mathrm{P}<0.05$. Error bars represent S.D. of biological replicates ( $\mathrm{n}=3$ or 4$)$. (F) Staining of leaf segments from BW-NIL (cer-ym.753) and Bowman with $0.05 \%(\mathrm{w} / \mathrm{v})$ toluidine blue solution at room temperature. Scale bar, $1 \mathrm{~cm}$.

BW-NIL (cer-ym.753) showed a similar degree of desiccation sensitivity to BW-NIL (cer-zv.268), which has previously been characterized as a desiccation sensitive mutant with impaired cutin deposition (Li et al. 2013). We found that the relative profiles of leaf cutin components of BWNIL (cer-ym.753) and BW-NIL (cer-zv.268) are essentially indistinguishable: the levels of $\omega \mathrm{OH}-9,10$ epoxy $\mathrm{C} 18$, 9(10), 16-OH C16, $\omega \mathrm{OH} \mathrm{C18:1} \mathrm{and} \omega \mathrm{OH} \mathrm{C} 16$, the four main leaf cutin monomers, were reduced by $91 \%, 78 \%, 70 \%$ and $63 \%$, respectively, in BW-NIL (cer-ym.753), and were reduced by approximately $92 \%, 82 \%, 72 \%$ and $61 \%$, respectively, in BW-NIL (cer-zv.268). Although the desiccation 


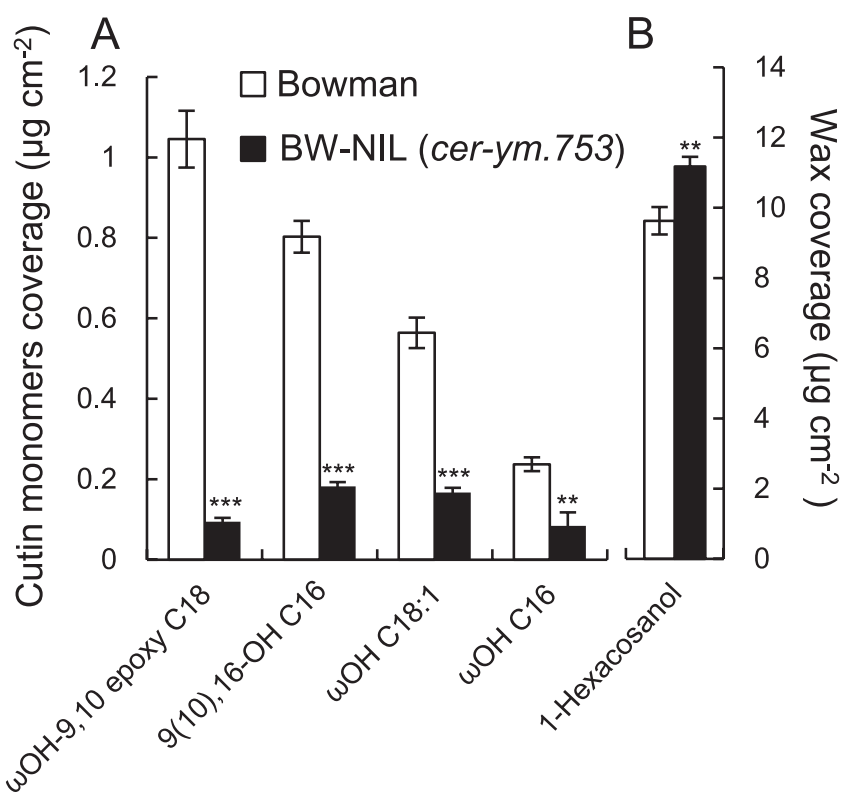

Fig. 2. Major cutin monomers (A) and wax component (B) of BWNIL (cer-ym.753) and Bowman leaves. Error bars represent S.D. of biological replicates $(n=4)$. Two and three asterisks denote significant differences of means at $\mathrm{P}<0.01$ and $\mathrm{P}<0.001$, respectively, between Bowman and the mutant as determined by a student's test.

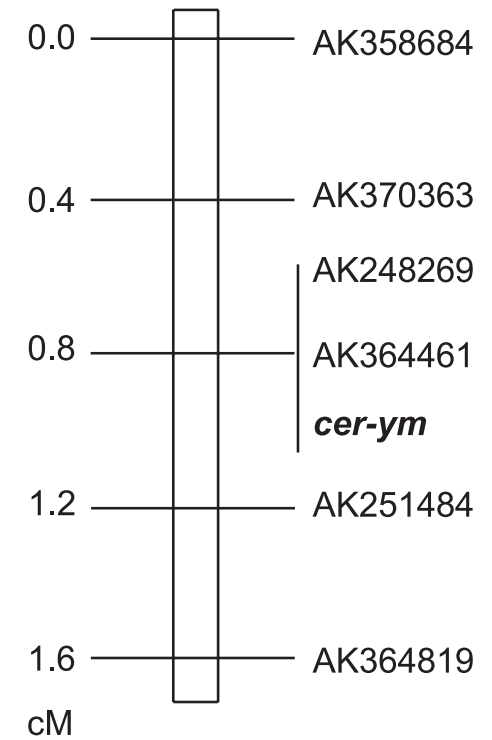

Fig. 3. Genetic map of the cer-ym locus. A set of 123 OUH602 $\times$ BWNIL (cer-ym.753) $\mathrm{F}_{2}$ progenies were analyzed with 6 genetic markers, which were previously positioned in pericentrometric region of chromosome 4H (Li et al. 2013). The genotype on cer-ym locus for each $\mathrm{F}_{2}$ line was deduced from the phenotypes of its $\mathrm{F}_{3}$ progenies.

sensitivity and cutin monomers contents were similar between $c e r-z v$ and cer-ym mutants, the former plants were weaker than the latter ones; most of the tillers were higher and stronger in cer-ym plants than in cer-zv plants (Supplemental Fig. 1). These results are consistent with that the $c e r-z v$ mutant shows more severe growth retardation than the cer-ym mutant (Franckowiak and Lundqvist 2012); cer-zv plants are semidwarf and very weak (BGS 439 in http://wheat.pw.usda.gov/ggpages/bgn/42/BGS478-571. $\mathrm{htm}$ ), while cer-ym plants are semidwarf and weak (BGS 526 in http://wheat.pw.usda.gov/ggpages/bgn/42/BGS572684.htm).

The association of cutin component contents or organization with desiccation sensibility is also found in cuticle mutants of other species. Arabidopsis lacs 2 mutant leaves exhibit marked reductions in both $\mathrm{C} 16$ and $\mathrm{C} 18$ cutin monomers, and leaf desiccation sensitivity is much higher in lacs 2-3 than in the wild type (Bessire et al. 2007, Weng et al. 2010). Arabidopsis $d c r$ mutant flowers display almost undetectable 9(10),16-dihydroxy-hexadecanoic acid, the main cutin monomer, and increased sensitivity to desiccation (Panikashvili et al. 2009). Tomato $c d l$ mutant shows largely decreased levels in all cutin monomers and severe symptoms of desiccation (Isaacson et al. 2009). Rice wdll mutant with no significant changes either in the composition and levels of waxes and cutin monomers shows a loose packing of the cuticle and increased rates of water loss (Park et al. 2010). Further studies are required to determine whether the cer-ym cuticle structure is also affected.

cer-ym mapped to a chromosome 4 H pericentromeric region cer-ym and cer-zv are two of the 79 eceriferum loci, which are confirmed by allelic tests (Lundqvist and Lundqvist 1988). Both the two mutant loci have been mapped to chromosome 4H (Druka et al. 2011, Li et al. 2013). In this current study, cer-ym were mapped co-segregated with AK364461, a marker that co-segregates with cer-zv, in the pericentromeric region where showed a very low recombinancy frequency (Mayer et al. 2011), suggesting an important role of this region for cuticle development.

\section{Acknowledgements}

We thank Professor Udda Lundqvist for constructive discussions. We thank National Small Grains CollectionAgricultural Research Service-U.S. Department of Agriculture for providing seeds. This research was supported by Key Project of Chinese National Programs for Fundamental Research and Development (973 Program, 2013CB429904 to G.C.), by Gansu Innovation Research Group Fund (1308RJIA002 to G.C.), by the National Natural Science Foundation of China (31170369 to G.C.), by the Genomics for Agricultural Innovation of the Ministry of Agriculture, Forestry, and Fisheries of Japan (TRS1002 to T.K.), by the Swiss National Science Foundation (grant 31003A-125009 to C.N.), as well as by a faculty exchange grant of the SinoSwiss Science and Technology Coorporation (grant EG15092011 to G.C. and C.N.).

\section{Literature Cited}

Bessire, M., C.Chassot, A.C. Jacquat, M.Humphry, S. Borel, J. MacDonald-ComberPetétot, J.P. Métraux and C.Nawrath (2007) 
A permeable cuticle in Arabidopsis leads to a strong resistance to Botrytis cinerea. EMBO J. 26: 2158-2168.

Boyer, J.S. (1982) Plant productivity and environment. Science 218 443-448.

Buschhaus, C. and R. Jetter (2011) Composition differences between epicuticular and intracuticular wax substructures: how do plants seal their epidermal surfaces? J. Exp. Bot. 62: 841-853.

Chen, G.X., T.Komatsuda, J.F. Ma, C.Nawrath, M. Pourkheirandish, A. Tagiri, Y.G.Hu, M.Sameri, X.Li, X.Zhao et al. (2011) An ATP-binding cassette subfamily $G$ full transporter is essential for the retention of leaf water in both wild barley and rice. Proc. Natl. Acad. Sci. USA 108: 12354-12359.

Druka,A., J.Franckowiak, U.Lundqvist, N. Bonar, J.Alexander, K. Houston, S.Radovic, F. Shahinnia, V.Vendramin, M. Morgante et al. (2011) Genetic dissection of barley morphology and development. Plant Physiol. 155: 617-627.

Franckowiak, J.D. (1997) Revised linkage maps for morphological markers in barley, Hordeum vulgare. Barley Genet. Newsl. 26: 9-21.

Franckowiak, J.D. and U.Lundqvist (2012) Descriptions of Barley Genetic Stocks for 2012. Barley Genet. Newsl. 42: 572-684.

Goodwin, S.M. and M. Jenks (2005) Plant cuticle function as a barrier to water loss. In: Jenks, M. and P.M. Hasegawa (eds.) Plant Abiotic Stress, Blackwell Publishing, Oxford, pp. 4-36.

Greer, S., M.Wen, D. Bird, X.Wu, L. Samuels, L. Kunst and R. Jetter (2007) The cytochrome P450 enzyme CYP96A15 is the midchain alkane hydroxylase responsible for formation of secondary alcohols and ketones in stem cuticular wax of Arabidopsis. Plant Physiol. 145: 653-667.

Heredia,A. (2003) Biophysical and biochemical characteristics of cutin, a plant barrier biopolymer. Biochim. Biophys. Acta 1620: $1-7$.

Isaacson, T., D.K.Kosma, A.J.Matas, G.J.Buda, Y.He, B.Yu, A.Pravitasari, J.D.Batteas, R.E.Stark, M.A.Jenks et al. (2009) Cutin deficiency in the tomato fruit cuticle consistently affects resistance to microbial infection and biomechanical properties, but not transpirational water loss. Plant J. 60: 363-377.

Jeffree,C.E. (1996) Structure and ontogeny of plant cuticles. In: Kerstiens, G. (ed.) Plant Cuticles: An Integrated Functional Approach, BIOS Scientific Publishers, Oxford, pp. 33-82.

Kannangara, R., C. Branigan, Y.Liu, T.Penfield, V.Rao, G. Mouille, H. Höfte, M.Pauly, J.L. Riechmann and P. Broun (2007) The transcription factor WIN1/SHN1 regulates cutin biosynthesis in Arabidopsis thaliana. Plant Cell 19: 1278-1294.

Kosambi,D.D. (1944) The estimation of map distances from recombi- nation values. Ann. Eugen. 12: 172-175.

Kosma, D.K., B. Bourdenx, A. Bernard, E.P.Parsons, S.Lü, J.Joubès and M.A. Jenks (2009) The impact of water deficiency on leaf cuticle lipids of Arabidopsis. Plant Physiol. 151: 1918-1929.

Lander, E.S., P. Green, J.Abrahamson, A. Barlow, M.J. Daly, S.E. Lincoln and L.A. Newberg (1987) MAPMAKER: an interactive computer package for constructing primary genetic linkage maps of experimental and natural populations. Genomics 1: 174-181.

Li, C., A.Wang, X.Ma, M.Pourkheirandish, S. Sakuma, N. Wang, S.Ning, E.Nevo, C.Nawrath, T.Komatsuda et al. (2013) An eceriferum locus, cer-zv, is associated with a defect in cutin responsible for water retention in barley (Hordeum vulgare) leaves. Theor. Appl. Genet. 126: 637-646.

Li, Y., F. Beisson, A.J.K.Koo, I. Molina, M.Pollard and J.Ohlrogge (2007) Identification of acyltransferases required for cutin biosynthesis and production of cutin with suberin-like monomers. Proc. Natl. Acad. Sci. USA 104: 18339-18344.

Lundqvist, U. and A.Lundqvist (1988) Mutagen specificity in barley for 1580 eceriferum mutants localized to 79 loci. Hereditas 108: $1-12$.

Mayer, K.F.X., M. Martis, P.E. Hedley, H. Simková, H. Liu, J.A. Morris, B. Steuernagel, S. Taudien, S. Roessner and H. Gundlach (2011) Unlocking the barley genome by chromosomal and comparative genomics. Plant Cell 23: 1249-1263.

Nawrath,C. (2006) Unraveling the complex network of cuticular structure and function. Curr. Opin. Plant Biol. 9: 281-287.

Niederl, S., T.Kirsch, M.Riederer and L.Schreiber (1998) Copermeability of ${ }^{3} \mathrm{H}$-labeled water and ${ }^{14} \mathrm{C}$-labeled organic acids across isolated plant cuticles: investigating cuticular paths of diffusion and predicting cuticular transpiration. Plant Physiol. 116: $117-123$.

Panikashvili,D., J.X. Shi, L.Schreiber and A.Aharoni (2009) The Arabidopsis DCR encoding a soluble BAHD acyltransferase is required for cutin polyester formation and seed hydration properties. Plant Physiol. 151: 1773-1789.

Park, J.J., P. Jin, J. Yoon, J.I. Yang, H.J.Jeong, K. Ranathunge, L. Schreiber, R. Franke, I.J.Lee and G.An (2010) Mutation in Wilted Dwarf and lethal 1 (WDL1) cause abnormal cuticle formation and rapid water loss in rice. Plant Mol. Biol. 74: 91-103.

Weng, H., I. Molina, J. Shockey and J.Browse (2010) Organ fusion and defective cuticle function in a lacs 1 lacs 2 double mutant of Arabidopsis. Planta 231: 1089-1100.

Yeats, T.H. and J.K.C. Rose (2013) The formation and function of plant cuticles. Plant Physiol. 163: 5-20. 